\title{
多能源耦合系统的运行优化与改进分析
}

\author{
张涛，朱䀐"，陈上，吴竺 \\ 同济大学机械与能源工程学院, 上海 201804 \\ *联系人, E-mail: zhu_tong@tongji.edu.cn \\ 2017-06-06 收稿, 2017-08-25 修回, 2017-08-28 接受, 2017-10-11 网络版发表 \\ 国家重点基础研究发展计划(2014CB249201)资助
}

\begin{abstract}
摘要建立含有光伏系统、传统天然气三联供能源系统、储能系统、热泉系统等的多能源耦合系统运行优化模 型. 考虑各个主要设备耗电量, 以运行费用最优为目标函数, 针对不同典型日负荷需求, 采用智能群优化算法获 得系统的运行参数, 进而分析研究多能源耦合系统的运行特性, 并在原能源系统的基础上, 针对原设计方案发电 容量较小, 分析研究如何进一步降低系统运行费用的改进方案。结果表明, 基于萤火虫优化算法构建的多能源耦 合系统的运行优化模型, 可较为准确发求解多能源耦合系统运行状态参数; 在能源系统原设计基础上, 通过增加 燃气内燃机机组的容量, 可以显著降低典型日能源费用; 制定合理的上网电价政策, 可进一步降低运行费用.
\end{abstract}

关键词＼cjkstart多能源耦合系统，运行优化，萤火虫优化算法，分布式能源系统

随着我国经济的快速发展，能源需求量不断增 大, 而我国一次能源以煤炭为主, 环境污染严重. 能 源、环境问题日益突出, 实现能源的清洁高效利用是 人们关注和研究的重点 ${ }^{[1,2]}$. 传统三联供分布式能源 系统由于使用清洁的一次能源且分散供能, 受到越 来越多的重视 ${ }^{[3]}$. 随着可再生能源的迅速发展, 分布 式能源系统耦合多种可再生能源, 为用户提供更为 清洁、环保、低碳的能源供应方案, 成为学者近年来 研究的重点.

分布式能源系统的发电主机一般包括内燃机、燃 气轮机、微型燃气轮机、燃料电池等; 余热回收利用 设备包括余热锅炉、换热器、溴化锂吸收式制冷机、 有机朗肯循环(ORC)余热发电系统等; 传统供能设备 包括燃气锅炉、螺杆式制冷机组等; 可再生能源设备 包括热百机组、光伏系统、光热系统等; 储能设备包 括冰(水)蓄冷罐、储热罐、蓄电池等. 显然, 多能源 耦合系统是一个复杂的多能源输人输出的系统, 包 含多种异质能源的同质化转换过程, 是能源流、物质
流和信息流交互的复杂能源系统.

随着多能源耦合系统的发展，人们相继提出能 源互联网 ${ }^{[4]}$ 、泛能网 ${ }^{[5]}$ 、微电网 ${ }^{[6]}$ 、微能源网 ${ }^{[7]}$ 、能 源枢纽 ${ }^{[8]}$ 、综合能源系统 ${ }^{[9]}$ 等概念, 能源系统的耦合 多种多样. 文献[10]针对含有太阳能、天然气、储能 的冷热电三联供系统, 建立优化协调模型, 以实例进 行计算, 结果表明清洁能源耦合互补发电优势明显。 文献[11]针对分布式能源系统与地源热洜系统各自 的优缺点, 建立了分布式能源系统和地源热葲的系 统耦合集成新方法, 结果表明多能源耦合系统可进 一步提高能源利用效率. 文献[12 15]针对耦合有机 朗肯循环的冷热电三联供系统开展研究, 获得影响 系统效率的关键参数，结果表明耦合系统适合某类 负荷需求且经济性较好.

多能源耦合系统的构建可以促进可再生能源的 消纳、提高能源利用效率，合理的多能源规划尤为重 要, 文献[16]针对多能源规划进行分析研究, 以各个 子系统为基础, 以电力系统为核心, 获得子系统的特 
异性, 充分发挥多种能源的互补特性. 文献[17]在考 虑电网和天然气网安全运行约束后, 提出以投资费 用和运行费用最小为目标的气网、电网耦合多阶段联 合规划模型.

多能源耦合系统包括电、热、气、冷等不同能源, 各个能源的转换、传输的特性各不相同, 如电能易于 传输、转换, 热能、冷能易于储存, 多能源网络耦合 互补非常重要. 文献[18]针对气网、电网进行稳态潮 流分析研究, 对气网、电网的联合调度方面开展研究, 获得模型中各个节点参数变化对网络潮流分布的影 响. 文献[19 21]研究热网、电网的耦合, 分别研究了 输送能源的延时、损耗等对多能源网络运行结果的影 响, 通过对储热环节的控制如何提高多能源耦合网 络的调节能力, 以及不同应用场景下储热装置的优 化设计方法、协调控制策略等.

以往针对传统分布式能源系统的运行研究多采 用固定的“以热定电”、“以电定热”等 ${ }^{[22,23]}$ 模式, 常把 运行优化问题简化的混合整数线性规划, 通过商业 软件如LINGO, Cplex, Gurobi等进行求解 ${ }^{[23,24]}$. 而针 对复杂的区域多能源耦合系统, 由于设备特性各异, 且系统复杂性高, 一般采用智能搜索优化算法进行 求解. 萤火虫优化算法是一种新颖的随机优化算法, 已经应用于多种工程优化和运行成本优化, 具有较 好的收玫速度和求解精度 ${ }^{[25]}$. 文献利用萤火虫算法 在分布式电源选址方面进行优化, 并与遗传算法比 较, 表明该算法具有收玫速度快、准确性高等特 点 ${ }^{[26,27]}$. 本文基于某区域多能源耦合系统为对象 (即 已知耦合系统的投建方案), 采用茧火虫优化算法, 仅以运行费用为优化目标建立相应的系统运行优化 模型; 在此基础上, 针对原设计方案发电容量较小, 分析研究通过系统改造, 如何进一步提高系统经济 性的改进方案.

\section{1 多能源耦合系统}

以位于青岛某多能源耦合系统为例, 该供能区域 总建筑面积7.26 $\times 10^{4} \mathrm{~m}^{2}$, 供热面积为 $5.14 \times 10^{4} \mathrm{~m}^{2}$. 实 际热负荷为 $4.82 \mathrm{MW}$, 实际冷负荷为 $4.03 \mathrm{MW}$. 用电 负荷为 $3.87 \mathrm{MW}$. 园区冬季采暖时间周期为 $141 \mathrm{~d}$, 夏 季制冷供能时间 $90 \mathrm{~d}$, 园区内天然气价格为 4 元 $/ \mathrm{Nm}^{3}$, 电力执行峰谷电价政策, 分为尖、峰、平、谷四种 电价, 具体如图 1所示. 峰谷电价及对应时间如图 1 所示.

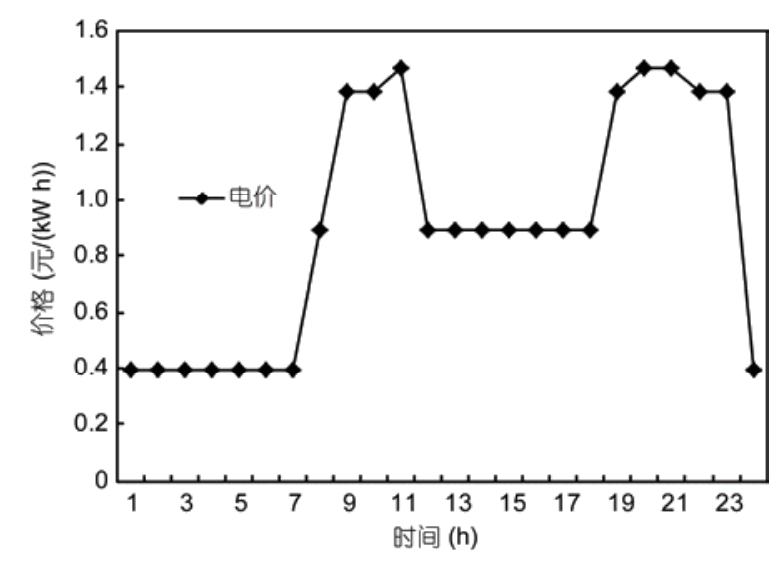

图 1 分时电价

Figure 1 Time-of-use electricity price

按照《燃气冷热电三联供工程技术规程》中民用 建筑发电容量一般可以取最大设计电力负荷的 $30 \%$ 规定, 为了保证发电机的利用小时数, 按照 $30 \%$ 的规 模来确定发电规模. 相比燃气轮机, 内燃机发电效率 随负载负荷的影响较小，从 $100 \%$ 负荷降到 $50 \%$ 负荷 时, 内燃机的发电效率从 $40 \%$ 变化到 $34 \%$ 左右, 为保 证内燃机在较高效率范围内运行, 本文优化限定内 燃机负荷率大于 $50 \%{ }^{[28]}$. 确定发电设备容量为 $2 \times$ $625 \mathrm{~kW}$, 区域内分布式能源系统发电采用并网不上 网，自发自用的运行模式. 同时，该区域设计安装9600 块光伏电池板, 单板功率为 $125 \mathrm{~W}$, 装机容量 $1.2 \mathrm{MW}$. 地源热百系统是可再生能源利用的一种重要方式, 且运行费用较低, 因此在系统运行时应该发挥最大 的能效, 不管是供冷还是供热, 该系统应该是一年中 运行时间最长的系统. 由于该项目的实施地点, 地下 情况较复杂, 地源热洜系统的投资较高. 地源热洜系 统的规模必须兼顾经济性和可再生能源的利用率等多 个指标, 故设计制冷负荷为 $350 \mathrm{~kW}$ 的地源热百系统.

本项目中天然气的价格按照 4 元 $/ \mathrm{Nm}^{3}$ 计算, 低 谷电价为 0.4 元 $/(\mathrm{kW} \mathrm{h})$, 综合考虑天然气热值、燃气 锅炉效率等因素, 在夜间低谷电期间燃气蓄热和电 加热蓄热的成本基本相同. 由于谷电期间电辅助加热 蓄热系统的经济性不明显, 因此不考虑蓄热供能. 一 般从经济性角度来看, 水蓄冷技术经济性要优于冰蓄 冷技术; 然而冰蓄冷的蓄冷密度一般为 40 50 $\mathrm{kW}$ $\mathrm{h} / \mathrm{m}^{3}$, 水蓄冷的蓄冷密度一般为 $7.0 \sim 11.6 \mathrm{~kW} \mathrm{~h} / \mathrm{m}^{3}$, 冰蓄冷槽的蓄冷密度是蓄冷水池蓄冷密度的 5 倍左 右, 相对水蓄冷而言冰蓄冷更节省空间, 可大幅度减 少土建工程量和费用, 且考虑项目存在尖、峰、平、 
谷电价差, 故系统考虑蓄冰桶为蓄能设备. 系统主要 由以下部分组成: 太阳能光伏系统、冷热电三联供系 统、水冷冷水机组系统、燃气锅炉系统、冰蓄冷系统、 地源热原系统等, 即多种能源耦合系统, 如图2所示.

综合以上因素, 项目采用多种能源耦合系统方 案: 太阳能光伏系统; 分布式能源系统的主要系统 配置为燃气内燃发电机冬季对接余热锅炉, 余热锅 炉产生的热水经板换供热, 夏天用溴化锂机组进行 制冷; 系统的基础制冷和基础制热负荷由地源热洜 系统负担; 调峰设备部分采用电空调+燃气锅炉; 同 时, 结合冰蓄冷满足最大冷热负荷需求, 设备参数信 息如表1所示.

\section{2 多能源耦合系统运行优化模型}

\section{1 主要设备优化模型}

光伏系统的发电效率主要受环境温度和太阳辐 射影响, 在太阳辐射强度不变的情况下, 环境温度升 高导致光伏电池板表面温度升高, 进而降低光伏系 统发电效率。计算公式如下:

$$
P_{\mathrm{PV}}=f_{\mathrm{PV}} P_{\text {cap }} \frac{I_{t}}{I_{\mathrm{STC}}}\left[1+\alpha\left(t_{\text {cell }}-t_{\text {cellsTC }}\right)\right],
$$

其中, $P_{\mathrm{PV}}$ 为光伏板发电功率; $f_{\mathrm{PV}}$ 为光伏阵列降额因 数, 取 $0.92 \sim 0.97 ; P_{\text {cap }}$ 为光伏板的额定容量, $\mathrm{kW} ; I_{t}$ 为 环境温度下太阳光照的辐射强度, $\mathrm{kW} / \mathrm{m}^{2} ; \alpha$ 为功率温 度系数, $-0.005 \% /{ }^{\circ} \mathrm{C} ; I_{\mathrm{STC}}$ 为标准测试条件下光照辐射 强度, $\mathrm{kW} / \mathrm{m}^{2} ; t_{\text {cellsTC }}$ 为标准测试条件下光伏电池温度, $25^{\circ} \mathrm{C} ; t_{\text {cell }}$ 为实际运行条件下光伏电池表面温度, ${ }^{\circ} \mathrm{C}$.
燃气进人内燃机后首先与空气混合并增压后, 经火花塞点火燃烧膨胀并推动活塞做功, 通过发电 机输出电能. 基于所选某内燃机设备的性能曲线 图, 拟合出内燃发电机部分负荷小的发电效率按下 式计算:

$$
\left\{\begin{array}{l}
E_{\mathrm{ICE}}=\eta_{\mathrm{ICE}} E_{\text {rated }}, \\
\eta_{\mathrm{ICE}}=-0.095 x^{2}+0.222 x+0.245,
\end{array}\right.
$$

其中, $E_{\mathrm{ICE}}$ 为内燃机发电功率, $\mathrm{kW} ; E_{\text {rated }}$ 为内燃机额 定发电功率, $\mathrm{kW} ; \eta_{\mathrm{ICE}}$ 为内燃机发电效率; $x$ 为内燃机 负荷率.

余热锅炉是连接燃气内燃机的重要设备, 本章 建立如下余热锅炉模型 ${ }^{[29]}$ :

$$
\left\{\begin{array}{l}
Q_{\mathrm{b}}=\eta_{\mathrm{b}} Q_{\mathrm{b}_{\text {_rated }},}, \\
\eta_{\mathrm{b}}=\eta_{\mathrm{b}_{\text {rated }}}\left(-0.6249 y^{2}+1.525 y+0.0951\right),
\end{array}\right.
$$

其中, $Q_{\mathrm{B}}$ 为余热锅炉供热量, $\mathrm{kW} ; Q_{\mathrm{b} \_ \text {rated }}$ 为余热锅炉 额定供热量, $\mathrm{kW} ; \eta_{\mathrm{b}}$ 为余热锅炉供热效率; $\eta_{\mathrm{b} \_ \text {rated }}$ 为余 热锅炉额定供热效率, 为 $0.85 ; y$ 为余热锅炉负荷率.

溴化锂制冷机组的热力学模型可为 ${ }^{[29]}$

$$
\left\{\begin{array}{l}
Q_{\mathrm{AC}}=C O P_{\mathrm{AC}} Q_{\mathrm{AR}}, \\
C O P_{\mathrm{AC}}=\frac{C O P_{\mathrm{AC} \_ \text {rated }} z}{0.024+1.266 z-0.928 z^{2}+0.638 z^{3}},
\end{array}\right.
$$

其中, $Q_{\mathrm{AC}}$ 为溴化锂制冷机组制冷功率, $\mathrm{kW} ; Q_{\mathrm{AR}}$ 为溴 化锂制冷机组额定制冷功率, $\mathrm{kW} ; C O P_{\mathrm{AC}}$ 为性能系 数; $C O P_{\mathrm{AC} \_ \text {rated }}$ 为额定性能系数, 为 $1.1^{[29]} ; z$ 为溴化锂 制冷机组负荷率.

螺杆式冷水机组是将电能转换成冷负荷, 热力 学模型可为

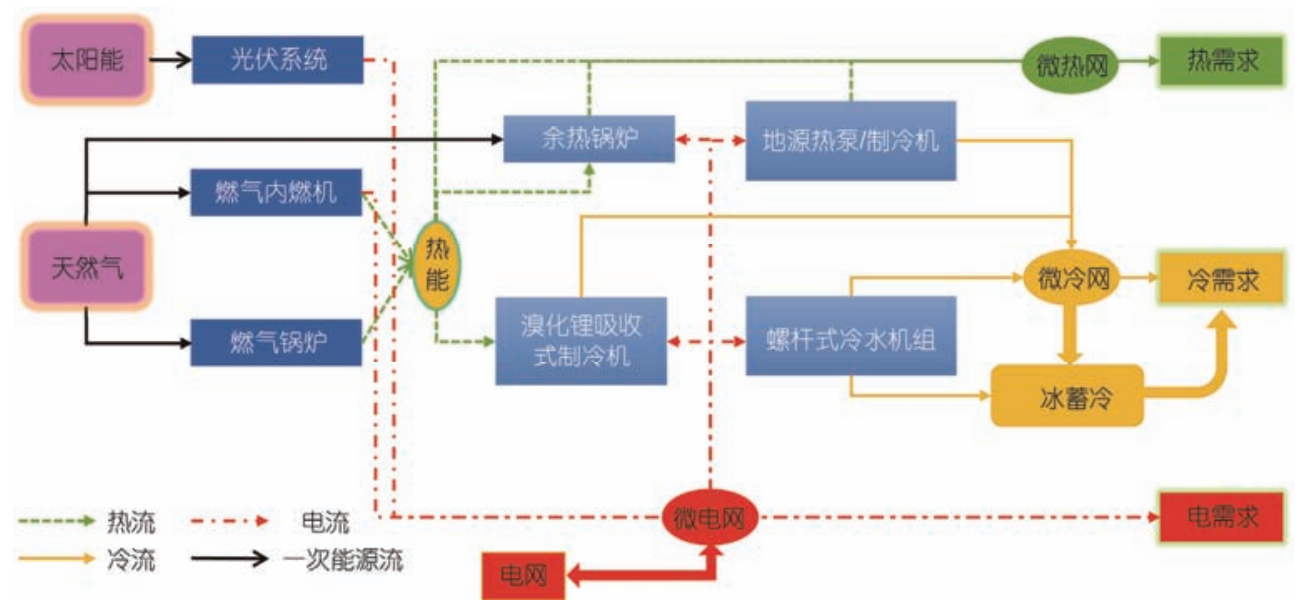

图 2 (网络版彩色)多能源耦合系统

Figure 2 (Color online) Multi-energy system 
表 1 主要设备参数信息

Table 1 Main equipment parameter information

\begin{tabular}{|c|c|c|c|}
\hline 设备名称 & \multicolumn{2}{|c|}{ 设备技术参数 } & 数量 \\
\hline & 发电功率 $(\mathrm{kW})$ & 625 & \\
\hline \multirow[t]{3}{*}{ 燃气内燃机 } & 发电效率 & $37.2 \%$ & 2台 \\
\hline & 总效率 & $87.4 \%$ & \\
\hline & 供热量(kW) & 1350 & \\
\hline \multirow[t]{4}{*}{ 余热锅炉 } & 运行工况 $\left({ }^{\circ} \mathrm{C}\right)$ & $68 / 130$ & 1台 \\
\hline & 配电功率 $(k V$ A) & 4.2 & \\
\hline & 制冷量(kW) & 350 & \\
\hline & 制冷输人功率 $(\mathrm{kW})$ & 65 & \\
\hline \multirow{5}{*}{ 地源热葲 } & 制冷运行工况 $\left({ }^{\circ} \mathrm{C}\right)$ & $7 / 12$ & $1 厶$ \\
\hline & 供热量(kW) & 403 & 坷 \\
\hline & 制热输人功率 $(\mathrm{kW})$ & 90 & \\
\hline & 制热运行工况 $\left({ }^{\circ} \mathrm{C}\right)$ & $50 / 40$ & \\
\hline & 制冷量(kW) & 2060 & \\
\hline \multirow[t]{3}{*}{ 螺杆式冷水机组 } & 运行工况 $\left({ }^{\circ} \mathrm{C}\right)$ & $7 / 14$ & 1台 \\
\hline & 输人功率 $(\mathrm{kW})$ & 223 & \\
\hline & 供热量(kW) & 3200 & \\
\hline \multirow[t]{3}{*}{ 燃气热水锅炉 } & 运行工况 $\left({ }^{\circ} \mathrm{C}\right)$ & $130 / 68$ & 1台 \\
\hline & 输人功率 $(\mathrm{kW})$ & 2 & \\
\hline & 蓄冰量(kW h) & 4800 & \\
\hline \multirow[t]{3}{*}{ 蓄冰桶 } & 蓄冰工况 $\left({ }^{\circ} \mathrm{C}\right)$ & $-3.8 / 0$ & 1套 \\
\hline & 释冰工况 $\left({ }^{\circ} \mathrm{C}\right)$ & $7 / 14$ & \\
\hline & 制冷量(kW) & 1176 & \\
\hline \multirow[t]{2}{*}{ 溴冷机 } & 配电功率(kW) & 30 & 1套 \\
\hline & 供冷工况参数 $\left({ }^{\circ} \mathrm{C}\right)$ & $7 / 14$ & \\
\hline
\end{tabular}

$$
Q_{\mathrm{EC}}=C O P_{\mathrm{EC}} Q_{\mathrm{ER}},
$$

其中, $Q_{\mathrm{EC}}$ 为螺杆式冷水机组制冷功率, $\mathrm{kW} ; Q_{\mathrm{ER}}$ 为螺 杆式冷水机组消耗的电功率, $\mathrm{kW} ; C O P_{\mathrm{EC}}$ 为性能系数, 为 5 .

$$
\begin{aligned}
& \text { 冰蓄冷储能剩余容量可表示为 } \\
& Q_{\text {store }}(k+1)=Q_{\text {store }}(k)\left(1-\mu_{\mathrm{Q} \_ \text {in }}\right)^{\Delta t}+Q_{\text {in }} \eta_{Q_{\text {_in }}}-\frac{Q_{\text {out }}}{\eta_{\text {Q_out }}},
\end{aligned}
$$

式中, $Q_{\text {store }}(k+1), Q_{\text {store }}(k)$ 分别为 $k+1$ 时刻、 $k$ 时刻的蓄

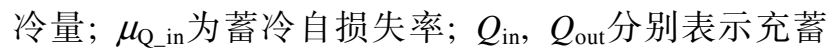
冷、释冷过程的冷量; $\eta_{\mathrm{Q} \_ \text {in }}$, $\eta_{\mathrm{Q} \_ \text {out }}$ 为与之对应的充放 能效率.

多能源耦合系统除了各个设备的数学模型约束 外, 还需要考虑整个系统的供能平衡约束, 如冷、热、 电负荷平衡约束:

$$
\begin{gathered}
E_{\text {need }}=E_{\text {Grid }}+E_{\mathrm{PV}}+\sum_{i=1}^{K} E_{\mathrm{ICE}, i}, \\
Q_{\mathrm{c} \_ \text {load }}=\sum_{i=1}^{n} Q_{c, i}, \\
Q_{\mathrm{h} \_ \text {load }}=\sum_{i=1}^{m} Q_{h, i},
\end{gathered}
$$

式中, $E_{\mathrm{Grid}}$ 为电网输人电能, $\mathrm{kW} ; E_{\mathrm{PV}}$ 为光伏发电系统 提供的电能, $\mathrm{kW} ; E_{\mathrm{ICE}, i}$ 为第 $i$ 类燃气内燃机组提供的 电能, $\mathrm{kW} ; Q_{\mathrm{c} \_ \text {load }}$ 为冷负荷, $\mathrm{kW} ; Q_{\mathrm{h} \_l o a d}$ 为热负荷, $\mathrm{kW}$; $Q_{\mathrm{c}, i}$ 为第 $i$ 个供冷设备提供的冷能, $\mathrm{kW} ; Q_{\mathrm{h}, i}$ 为第 $i$ 个供 热设备提供的热能, $\mathrm{kW}$.

考虑到各个设备的运行工况，设置所有设备的 运行负荷率不低于 $50 \%$, 当负荷率低于 $50 \%$ 时, 该设 备停机. 则供能设备容量约束为

$$
0.5 P_{i_{\text {_rated }}} \leqslant P_{i} \leqslant P_{i_{\text {r rated }}},
$$

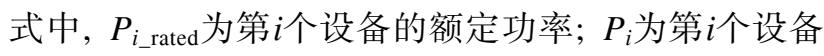
的实际输出功率.

针对储能设备存在如下约束:

$$
\begin{aligned}
& P_{\mathrm{s}_{-} \text {min }} \leqslant P_{\mathrm{s}}^{t} \leqslant P_{\mathrm{s}_{-} \text {max }}, \\
& P_{\mathrm{b}_{-} \text {min }} \leqslant P_{\mathrm{b}}^{t} \leqslant P_{\mathrm{b}_{-} \text {max }},
\end{aligned}
$$

式中, $P_{\mathrm{s}_{-} \min }, P_{\mathrm{s}_{-} \max }$ 分别为储能设备的最小、最大容量; $P_{\mathrm{b}_{-} \min }, P_{\mathrm{b}_{-} \max }$ 分别为储能设备的最小、最大充放能功 率.

在考虑各个设备自用电的情况, 建立目标函数 为典型日的能源费用最低，如：

$$
\begin{aligned}
\min \cos t= & \sum_{i=1}^{24}\left(e(i) \cdot Q_{\mathrm{buy}}(i)+g a s \cdot V_{\text {gas }}(i)\right) \\
& -\sum_{i=1}^{24}\left(e(i) \cdot e_{\text {ratio }} \cdot Q_{\text {sell }}(i)\right),
\end{aligned}
$$

式中, cost为典型日能源费用, 万元; $e(i)$ 为第 $i$ 小时的 电价, 元 $/(\mathrm{kW} \mathrm{h}) ; Q_{\text {buy }}(i)$ 为第 $i$ 小时的购电电量, $\times 10^{4}$ $\mathrm{kW} \mathrm{h} ;$ gas 为天然气价格, 元 $/(\mathrm{kW} \mathrm{h}) ; V_{\text {gas }}(i)$ 为第 $i$ 小时 购气的热量, $\times 10^{4} \mathrm{~kW} \mathrm{~h} ; Q_{\text {sell }}(i)$ 为第 $i$ 小时的上网电量, $\times 10^{4} \mathrm{~kW} \mathrm{~h} ; e_{\text {ratio }}$ 为网电价比.

系统设备增容的投资回收期计算公式如下:

$$
S P T=\Delta \text { Invest }_{\text {inc }} / C_{\text {red }},
$$

式中, $S P T$ 为系统增容费的静态投资回收期; $\Delta$ Invest $_{\mathrm{inc}}$ 为系统增容后投资增加额, 万元; $C_{\text {red }}$ 为系统增容运 行费用年节约额, 万元/年.

\section{2 独火虫优化算法}

萤火虫算法(firefly algorithm, FA)是一种基于生 
物群智能的随机优化算法, 源于自然界萤火虫发光 求偶的行为研究, 把每个个体作为搜索空间中的一 个微粒, 微粒基于搜索空间移动搜索, 微粒的运动基 于周围微粒所发出的苂光强度和光强吸收系数决定, 微粒向苂光素值高的位置移动, 苂光素值对应适应 度函数值, 因此微粒通过在动态决策域内寻找最高 苂光素值的位置而确定适应度函数的最优值.

显然茧火虫算法包含两个要素, 即亮度和吸引 度. 亮度体现了萤火虫所处位置的优劣并决定其移 动方向, 吸引度决定了萤火虫移动的距离, 通过亮度 和吸引度的不断更新, 从而实现目标优化. 萤火虫的 相对苂光亮度为

$$
I=I_{0} \times \mathrm{e}^{-\gamma r_{i j}},
$$

其中, $I_{0}$ 为萤火虫的最大萤光亮度, 即自身 $(r=0$ 处) 苂 光亮度, 与目标函数值相关, 目标函数值越优自身亮 度越高; $\gamma$ 为光强吸收系数, 可设为常数; $r_{i j}$ 为萤火虫 $i$ 与 $j$ 之间的空间距离, 在求解离散问题时, 距离可根 据实际情况定义为

$$
\beta=\beta_{0} \cdot \mathrm{e}^{-\gamma} r_{i j}=\left\|x_{i}-x_{j}\right\|=\sqrt{\sum_{k=1}^{d}\left(x_{i, k}-x_{j, k}\right)},
$$

其中, $d$ 为空间维数; $x_{i, k}$ 为萤火虫 $i$ 在 $d$ 维空间中的第 $k$ 个分量.

萤火虫的吸引度为

$$
\beta=\beta_{0} \times \mathrm{e}^{-\gamma r^{2}{ }^{2}},
$$

其中, $\beta_{0}$ 为最大吸引度, 即光源处 $(r=0$ 处) 的吸引度; 因为苂光会随着距离的增加和传播媒介的吸收逐渐 减弱, 所以设置光强吸收系数以体现此特性, 可设为 常数.

茧火虫 $i$ 被吸引向茧火虫 $j$ 移动的位置更新由下式 计算:

$$
x_{i}=x_{i}+\beta \times\left(x_{j}-x_{i}\right)+\alpha \times(\text { rand }-1 / 2),
$$

其中, $x_{i}, x_{j}$ 为萤火虫 $i$ 与 $j$ 所处的空间位置; $\alpha$ 为步长因 子, 是 $[0,1]$ 上的常数; $\operatorname{rand}$ 为 $[0,1]$ 上服从均匀分布的 随机因子.

光强吸收系数 $\gamma$ 对吸引度 $\beta$ 影响较大, 决定了茧 火虫个体的移动跨度大小和收玫速度等. 当两个茧 火虫之间的距离较小时, 吸引度函数展开如下:

$$
\beta=\beta_{0} \times \mathrm{e}^{-\gamma r^{2}{ }^{2}}=\beta_{0}\left(1-\gamma r^{2}{ }_{i j}+0.5 \gamma^{2} r^{4}{ }_{i j}+\cdots\right) .
$$

若光强吸收系数接近零, 则吸引度趋近于 $\beta_{0}$, 则 位置更新公式:

$$
x_{i}=x_{i}+\beta_{0} \cdot\left(x_{j}-x_{i}\right)+\alpha \cdot(\text { rand }-1 / 2) .
$$

若 $\beta_{0}$ 为 1 时, 则位置更新公式:

$$
x_{i}=x_{j}+\alpha \times(\text { rand }-1 / 2) .
$$

即萤火虫所发光在空间中无任何衰减，茧火虫 均可在所处范围内直接找到最优、最亮个体.

假如光强吸收系数 $\gamma$ 较大时, 则 $\beta$ 也取值较大, 位置更新公式为

$$
x_{i}=x_{i}+\alpha \times(\text { rand }-1 / 2) .
$$

步长因子 $\alpha$ 可以提高个体的差异性，扩展算法的 搜索能力, 避免过早收玫, 取值应与搜索区间的范 围、维数相关.

茧火虫优化算法具有简单易懂、参数少和易实现 等优点, 已经在诸多领域取得了较好的应用. 本文即 采用该方法优化计算，流程图如图3所示.

\section{3 结果分析}

\section{1 多能源耦合系统运行优化结果}

针对该多能源耦合系统，如图4为典型日的冷、 热、电负荷需求，系统设计为并网不上网的方式运行， 发电仅承担基本电负荷, 不足部分由市电补充. 总体 来说，燃机运行的时段在满足供冷供热要求的前提 下，应该避开市政谷电的时间.在夜间低谷电价期 间，尽量不开启燃机。

采用Matlab编写计算模型，茧火虫优化算法初 始设置种群规模为 50 ; 最大迭代次数 200 . 优化结果

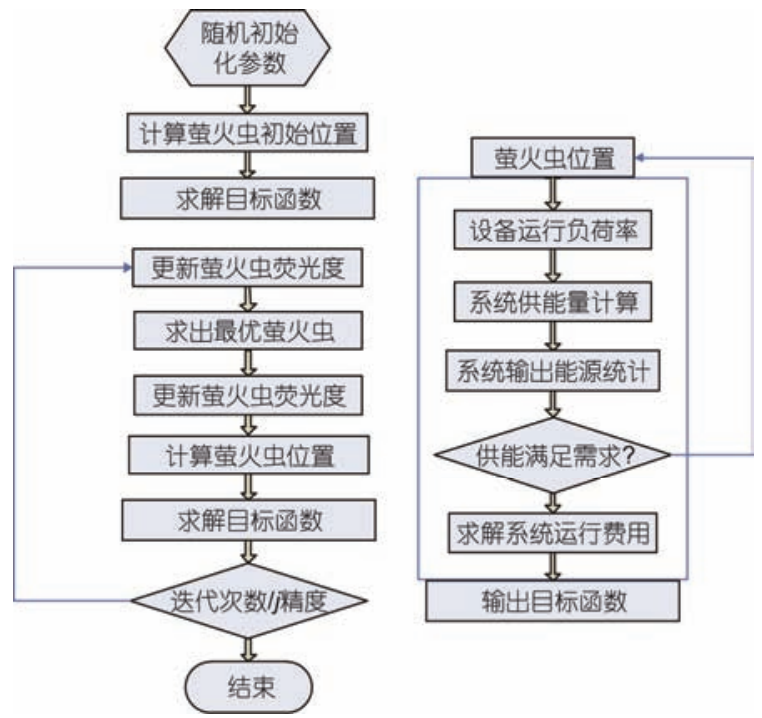

图 3 (网络版彩色) 茧火虫算法流程图

Figure 3 (Color online) Flowchart of firefly algorithm 

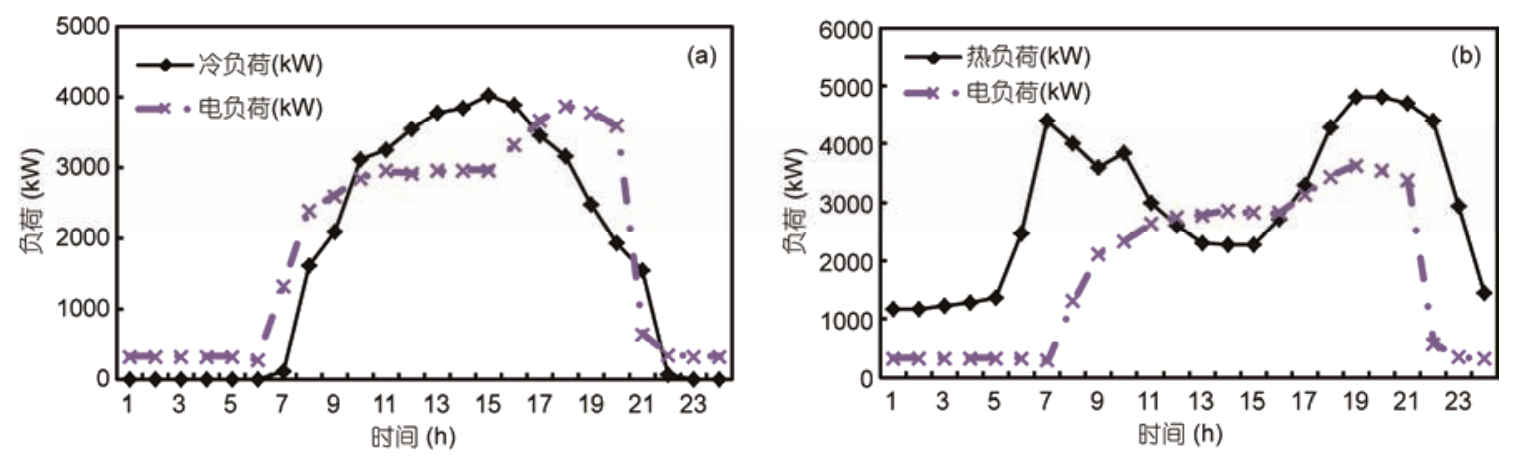

图 4 (网络版彩色)典型日冷(热)电负荷. (a) 夏季典型日; (b) 冬季典型日

Figure 4 (Color online) Loads of typical days. (a) Summer day; (b) winter day

表明迭代次数在 120 次左右收玫, 显然该算法可较为 准确地求解多能源耦合系统运行优化.

如图5所示优化计算夏季典型日设备供冷(电)出 力情况, 供冷出力主要包括螺杆机供冷、地源热泉供 冷、冰蓄冷供冷、溴冷机供冷. 可知夏季供冷, 主要 依靠螺杆式压缩式制冷机和燃气内燃机组耦合的溴 冷机. 蓄冰桶夜间蓄冷, 可显著降低螺杆机组的最大 负荷. 其中供电出力设备耗电为负值, 多能源耦合系 统主要耗电包括: 夜间蓄冷耗电、白天螺杆机制冷耗 电、地源热洜耗电、澳冷机耗电. 电力来源主要来 自市网电力、内燃机发电、光伏发电, 其中购买市电 较多.

如图6所示夏季典型日能源费用, 由于该项目设 计阶段天然气气价较高, 为兼顾项目的清洁绿色和 经济合理, 故选择内燃机容量较小, 仅为 $1250 \mathrm{~kW}$, 光伏为 $1200 \mathrm{~kW}$, 根据图 5 的出力情况, 优化计算出 逐时所需能源的费用. 可知: 夏季逐时费用最高出现 19:00 20: 00, 主要因为这个时间冷负荷、电负荷需

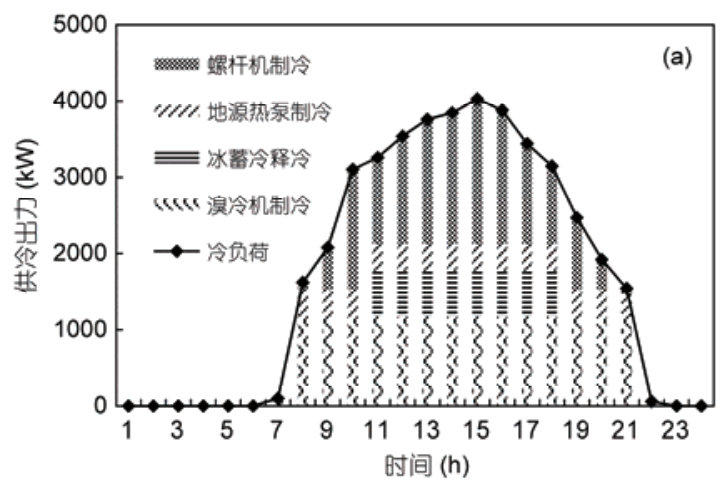

求较大, 且该时间段为峰值电价. 夏季典型日的天然 气使用费用为 $1.88 \times 10^{4}$ 元, 购电费用为 $2.60 \times 10^{4}$ 元, 总能源费用为 $4.48 \times 10^{4}$ 元.

如图7所示冬季典型日设备供热(电)出力. 由于 冬季全天 $24 \mathrm{~h}$ 需要供暖, 故地源热泉作为可再生供 能, 全天 $24 \mathrm{~h}$ 运行. 由于内燃机容量设计较小, 与内 燃机耦合的余热锅炉供热出力并不高, 主要热负荷 由燃气锅炉提供; 其中设备耗电为负值, 地源热原全 天24 h运行均消耗电能. 电力来源主要来自市网电 力、内燃机发电、光伏发电.

如图8所示冬季典型日能源费用. 由于冬季燃气 锅炉供暖耗气量较大, 故燃气费用较大. 冬季典型日 的天然气使用费用为 $3.85 \times 10^{4}$ 元，购电费用为 $2.18 \times$ $10^{4}$ 元, 总能源费用为 $6.03 \times 10^{4}$ 元.

按照系统设计机组运行时间安排(传统运行方 法): 供冷季燃机运行时间为每天早 7:00到晚 22:00, 每天共计 $16 \mathrm{~h}$, 整个供冷季燃机运行小时数为 $1440 \mathrm{~h}$. 供热季燃机运行时间按照每天早7:00到晚22:00, 每天

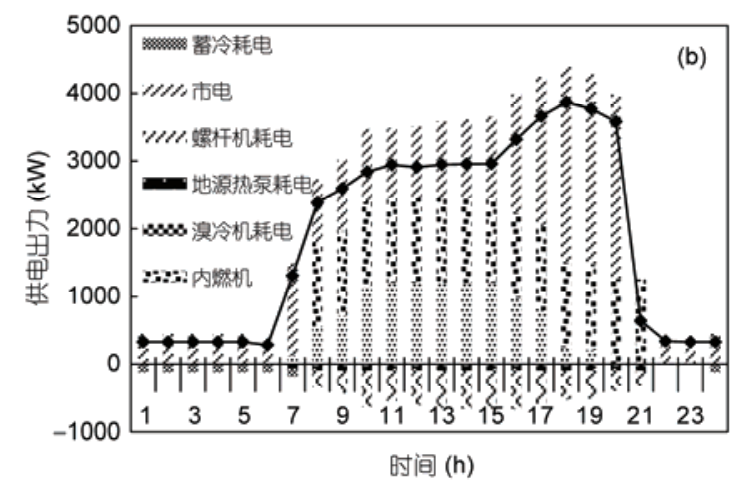

图 5 夏季典型日设备供冷(电)出力. (a) 供冷出力; (b) 供电出力

Figure 5 Equipment outputs of typical day in summer. (a) Cooling outputs; (b) electric outputs 


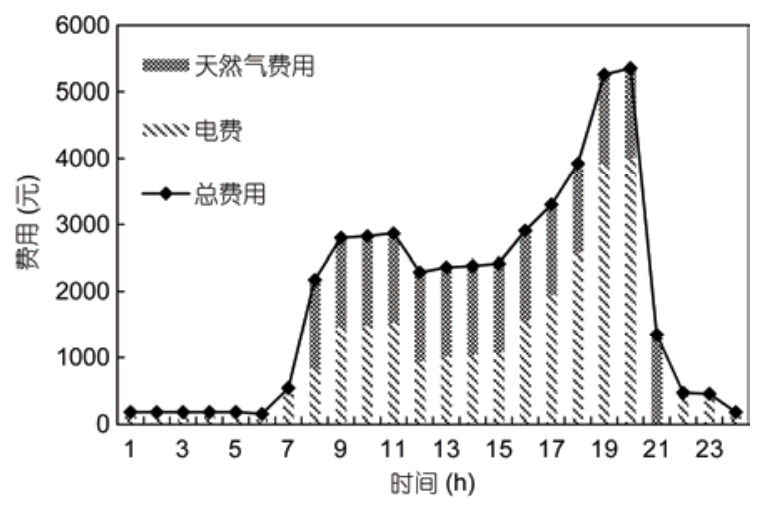

图 6 夏季典型日能源费用

Figure 6 Energy costs of typical day in summer
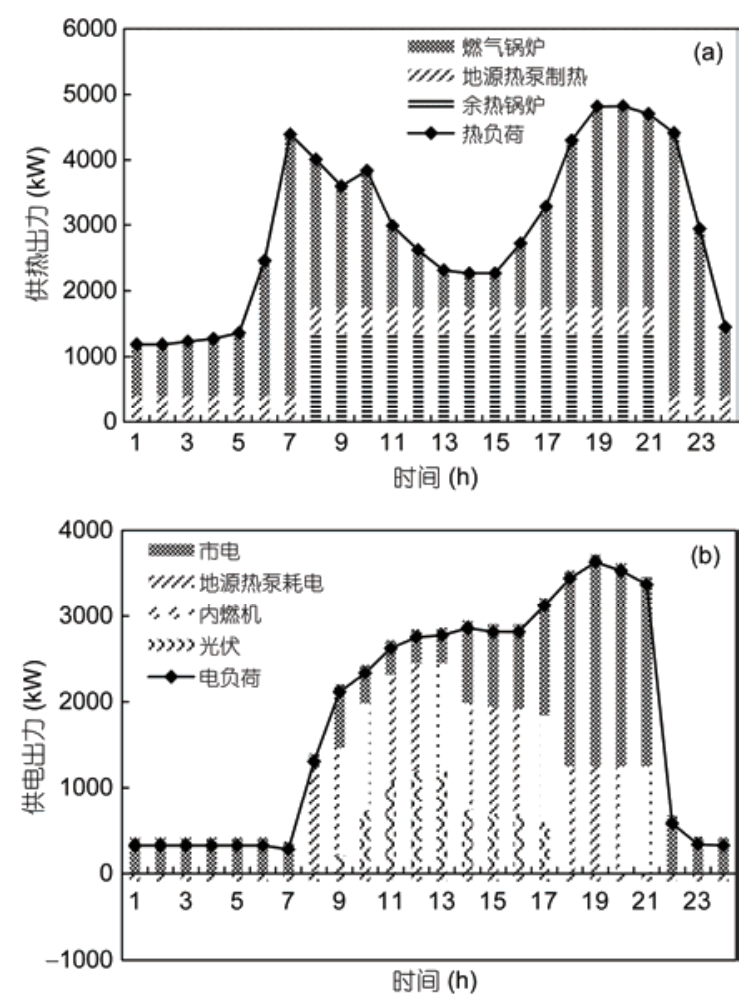

图 7 冬季典型日设备供热(电)出力. (a) 供热出力; (b) 供电出力

Figure 7 Equipment outputs of typical day in winter. (a) Heating outputs; (b) electric outputs

共计 $16 \mathrm{~h}$ 考虑, 整个供热季燃机运行小时数为 $2256 \mathrm{~h}$, 全年燃机利用小时数为 $3696 \mathrm{~h}$. 以此计算典型日的运 行费用如表2所示总费用. 通过优化计算的典型日的 能源费用分别由 $4.81 \times 10^{4}, 6.23 \times 10^{4}$ 元降到 $4.48 \times 10^{4}$, $6.03 \times 10^{4}$ 元.

\section{2 多能源耦合系统改进分析}

如上介绍的多能源耦合系统按照天然气 4 元 $/ \mathrm{Nm}^{3}$

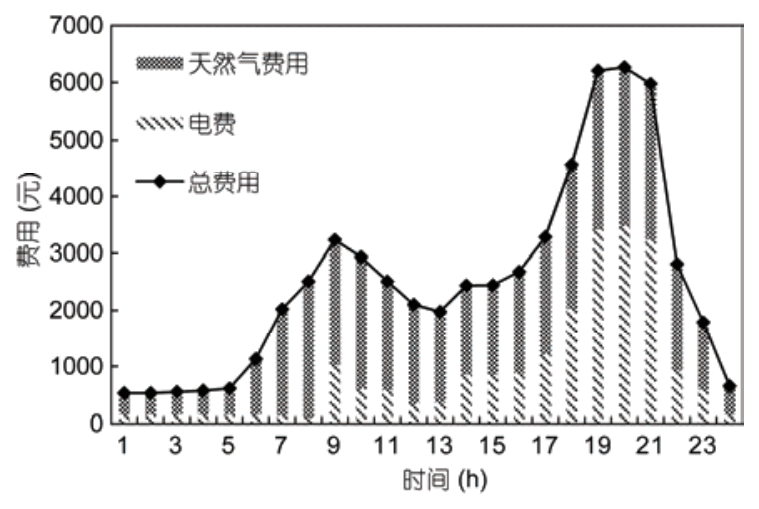

图 8 冬季典型日能源费用

Figure 8 Energy costs of typical day in winter

表 2 不同容量内燃机对应系统典型日能源费用(万元)

Table 2 Energy costs of typical day with different ICE capacities

\begin{tabular}{llccc}
\hline 典型日 & 策略 & 电费 & 天然气费用 & 总费用 \\
\hline \multirow{2}{*}{ 夏季典型日 } & 传统运行方法 & 2.85 & 1.96 & 4.81 \\
& 优化运行策略 & 2.60 & 1.88 & 4.48 \\
\multirow{2}{*}{ 冬季典型日 } & 传统运行方法 & 2.27 & 4.02 & 6.29 \\
& 优化运行策略 & 2.18 & 3.85 & 6.03 \\
\hline
\end{tabular}

的价格设计而得, 为兼顾清洁能源的利用和经济性 的考虑, 设计燃气内燃机的容量偏小, 仅为 $1250 \mathrm{~kW}$. 在原系统的基础上, 考虑到某内燃机组品牌的额定 输出功率范围, 选取内燃机容量为 $2000 \mathrm{~kW}$ (选取两 台额定功率为 $1000 \mathrm{~kW}$ 的机组)、 $2500 \mathrm{~kW}$ (选取两台额 定功率为 $1250 \mathrm{~kW}$ 的机组), 能源站多余电力可以上 网, 上网电价为同时购电价格的 $80 \%$ (即上网电价比 为 0.8$)$, 对系统进行运行优化求解.

当内燃机容量为 $2000 \mathrm{~kW}$ 的夏季典型日计算结 果, 随着内燃机容量的增加, 可知与内燃机耦合的溴 冷机的供冷量和余热锅炉的供热量相应增加. 总体 来看, 内燃机发电量和光伏系统发电量之和仅仅超 出电负荷一点, 对系统的影响并不大.

当内燃机容量为 $2500 \mathrm{~kW}$ 时，将会有更多的多余 电量, 假设设置上网电价比为 0.8 , 优化计算结果如 图9所示， 8 时 15 时之间，将会有大量的电量多余, 进而可以通过上网电价获得部分收益. 其中售电收 益设为负值.

如表3所示经济分析，内燃机扩容到 $2000 \mathrm{~kW}$ 时, 年运行费用为 $1177.1 \times 10^{4}$ 元，相比现有设计方案可降 低年运行费用 $6.1 \%$. 当进一步扩容到 $2500 \mathrm{~kW}$ 时，且 
表 3 不同容量内燃机对应系统年能源费用 (万元)

Table 3 Energy costs of typical day with different ICE capacities

\begin{tabular}{ccccccc}
\hline 内燃机容量 $(\mathrm{kW})$ & 上网电价比 & 电费 & 天然气费用 & 售电收益 & \multicolumn{2}{c}{ 总费用 } \\
\hline 1250 & 无 & 541.4 & 712.1 & 0 & 1253.4 \\
2000 & 无 & 296.2 & 880.9 & 0 & 1177.1 \\
2500 & 0.8 & 206.8 & 1017.7 & -112.1 & 1112.3 \\
\hline
\end{tabular}

存在上网电价时, 显然市网购电量下降很多, 而然气 消耗费用增加较多, 同时存在一小部分的上网收益. 优化结果显示: 该情况下年运行费用为 $1112.3 \times 10^{4}$ 元, 相比内燃机容量为 $1250 \mathrm{~kW}$ 的系统, 费用减少可达 $11.3 \%$. 年节约费用约 $141 \times 10^{4}$ 元, 通过计算内燃机 增容费用的静态投资回收年限为 4.43 年, 显然, 根据 市场现有内燃机组机型, 适当扩容内燃机容量, 通过 制定上网电价政策, 可以大幅度降低系统的运行费用.

\section{4 结论}

本文以某多能源耦合系统案例为研究对象, 建 立系统各个设备优化模型, 基于茧火虫优化算法, 构 建基于智能算法的多能源耦合系统运行优化模型, 进 而分析研究多能源耦合系统的运行特性, 并在原能源
系统的基础上, 研究改进的方法, 可得到以下结论.

基于萤火虫优化算法, 构建多能源耦合系统的 运行优化模型, 该方法可以较为准确地求解多能源 耦合系统运行状态参数.

该多能源耦合系统原设计方法为兼顾清洁能源 的利用和经济性的考虑, 设计燃气内燃机的容量偏 小, 本文得出: 当容量取为 $2000 \mathrm{~kW}$ 时, 相比内燃机 容量为 $1250 \mathrm{~kW}$ 的系统, 年费用减少了 $6.1 \%$; 当容量 取为 $2500 \mathrm{~kW}$ 时, 融人一定的上网电价比, 年费用可 降低 $11.3 \%$.

在能源系统原设计基础上, 通过增加燃气内燃 机机组的容量, 可以显著降低典型日能源费用; 政府 制定合理的上网电价政策，如设置合理的上网电价 比，有利于节约能源，提高系统的经济性.

\section{参考文献}

1 Wu J Z. Drivers and state-of-the-art integrated energy systems in Europe (in Chinese). Automat Elect Power Syst, 2016, 5: 1-7 [吴建中. 欧洲综合能源系统发展的驱动与现状. 电力系统自动化, 2016, 5: 1-7]

2 Chen H Y, Wen J Z, Wang Z Y, et al. Transfer laws and equations of energy networks (in Chinese). J Xi'an Jiaotong Univ, 2014, 10: 66-76 [陈皓勇, 文俊中, 王增显, 等. 能量网络的传递规律与网络方程. 西安交通大学学报, 2014, 10: 66-76]

3 Zhang T, Zhu T, Gao N P, et al. Optimization design and multi-criteria comprehensive evaluation method of combined cooling heating and power system (in Chinese). Proc CSEE, 2015, 35: 3706-3713 [张涛, 朱粀, 高乃平, 等. 分布式冷热电能源系统优化设计及多指 标综合评价方法的研究. 中国电机工程学报, 2015, 35: 3706-3713]

4 Huang A Q, Crow M L, Heydt G T, et al. The future renewable electric energy delivery and management (FREEDM) system: The energy internet. Proc IEEE, 2011, 99: 133-148

5 Gan Z X, Zhu X J, Wang C, et al. Ubiquitous energy internet-new energy internet coupling with information and energy (in Chinese). Eng Sci, 2015, 9: 98-104 [甘中学, 朱晓军, 王成, 等. 泛能网一信息与能量耦合的能源互联网. 中国工程科学, 2015, 9: 98-104]

6 Jin M, Feng W, Liu P, et al. MOD-DR: Microgrid optimal dispatch with demand response. Appl Energy, 2017, 187: 758-776

7 Tian S M, Luan W P, Zhang D X, et al. Technical forms and key technologies on energy internet (in Chinese). Proc CSEE, 2015, 35: 3482-3494 [田世明, 奕文鹏, 张东霞, 等. 能源互联网技术形态与关键技术. 中国电机工程学报, 2015, 14: 3482-3494]

8 Rastegar M, Fotuhi-Firuzabad M, Lehtonen M. Home load management in a residential energy hub. Elect Power Syst Res, 2015, 119: 322-328

9 Jiang X S, Jing Z X, Li Y Z, et al. Modelling and operation optimization of an integrated energy based direct district water-heating system. Energy, 2014, 64: 375-388

10 Xiong Y, Wu J K, Wang Q, et al. An optimization coordination model and solution for combined cooling, heating and electric power systems with complimentary generation of wind, PV, gas and energy storage (in Chinese). Proc CSEE, 2015, 35: 3616-3625 [熊焰, 吴杰 康, 王强, 等. 风光气储互补发电的冷热电联供优化协调模型及求解方法. 中国电机工程学报, 2015, 35: 3616-3625] 
11 Kang S S, Li H Q, Cai B, et al. Research on multi-energy supply system of distributed energy system coupled with ground source heat pump (in Chinese). J Eng Thermophys, 2013, 5: 817-821 [康书硕, 李洪强, 蔡博, 等. 分布式能源系统与地源热㫤耦合的系统研究. 工程热物理学报, 2013, 5: 817-821]

12 Mohammadi A, Kasaeian A, Pourfayaz F, et al. Thermodynamic analysis of a combined gas turbine, ORC cycle and absorption refrigeration for a CCHP system. Appl Thermal Eng, 2017, 111: 397-406

13 Ebrahimi M, Ahookhosh K. Integrated energy-exergy optimization of a novel micro-CCHP cycle based on MGT-ORC and steam ejector refrigerator. Appl Thermal Eng, 2016, 102: 1206-1218

14 Hajabdollahi H. Investigating the effects of load demands on selection of optimum CCHP-ORC plant. Appl Thermal Eng, 2015, 87: $547-558$

15 Fang F, Wei L, Liu J, et al. Complementary configuration and operation of a CCHP-ORC system. Energy, 2012, 46: 211-220

16 Shao C C, Wang X F, Wang X L, et al. Probe into analysis and planning of multi-energy systems (in Chinese). Proc CSEE, 2016, 14: 3817-3829 [邵成成，王锡凡，王秀丽，等. 多能源系统分析规划初探. 中国电机工程学报, 2016, 14: 3817-3829]

$17 \mathrm{Hu} \mathrm{Y,} \mathrm{Bie} \mathrm{Z} \mathrm{H,} \mathrm{Li} \mathrm{G} \mathrm{F,} \mathrm{et} \mathrm{al.} \mathrm{Integrated} \mathrm{planning} \mathrm{of} \mathrm{natural} \mathrm{gas} \mathrm{network} \mathrm{and} \mathrm{composite} \mathrm{power} \mathrm{system} \mathrm{(in} \mathrm{Chinese).} \mathrm{Proc} \mathrm{CSEE,} \mathrm{2017,} \mathrm{1:}$ 45-54 [胡源, 别朝红, 李更丰, 等. 天然气网络和电源、电网联合规划的方法研究. 中国电机工程学报, 2017, 1: 45-54]

18 Martinez-Mares A, Fuerte-Esquivel C R. A unified gas and power flow analysis in natural gas and electricity coupled networks. IEEE Trans Power Syst, 2012, 27: 2156-2166

19 Chen L, Xu F, Wang X, et al. Implementation and effect of thermal storage in improving wind power accommodation (in Chinese). Proc CSEE, 2015, 17: 4283-4290 [陈磊, 徐飞, 王晓, 等. 储热提升风电消纳能力的实施方式及效果分析. 中国电机工程学报, 2015, 17: 4283-4290]

20 Gu Z P, Kang C Q, Chen X Y, et al. Operation optimization of integrated power and heat energy systems and the benefit on wind power accommodation considering heating network constraints (in Chinese). Proc CSEE, 2015, 14: 3596-3604 [顾泽鹏，康重庆，陈新宇，等. 考虑热网约束的电热能源集成系统运行优化及其风电消纳效益分析. 中国电机工程学报, 2015, 14: 3596-3604]

21 Xu F, Min Y, Chen L, et al. Combined electricity-heat operation system containing large capacity thermal energy storage (in Chinese). Proc CSEE, 2014，29：5063-5072 [徐飞, 闵勇, 陈磊, 等. 包含大容量储热的电-热联合系统. 中国电机工程学报, 2014, 29: 5063-5072]

22 Yang Y, Zhang S, Xiao Y. An MILP (mixed integer linear programming) model for optimal design of district-scale distributed energy resource systems. Energy, 2015, 90: 1901-1915

$23 \mathrm{Li} \mathrm{L}, \mathrm{Mu} \mathrm{H}, \mathrm{Li} \mathrm{N}$, et al. Economic and environmental optimization for distributed energy resource systems coupled with district energy networks. Energy, 2016, 109: 947-960

24 Akbari K, Jolai F, Ghaderi S F. Optimal design of distributed energy system in a neighborhood under uncertainty. Energy, 2016, 116: $567-582$

25 Xu B J, Wang Y N. Optimizing the composite cost of electric vehicles based on the firefly optimization model (in Chinese). CAAI Trans Intell Syst, 2017, 2: 166-171 [许斌杰, 王耀南. 茧火虫算法的电动汽车综合成本运行优化研究. 智能系统学报, 2017, 2: 166-171]

26 Chen J J, Jiang T Z, Zhou Y, et al. Reasonable allocation of distributed generation in distribution system considering voltage sag (in Chinese). Power Syst Tech, 2014, 8: 2244-2249 [陈家俊, 蒋铁铮, 周勇, 等. 考虑电压骤降的分布式电源定容和选址. 电网技术, 2014, 8: 2244-2249]

27 Sulaiman M H, Mustafa M W, Azmi A, et al. Optimal allocation and sizing of distributed generation in distribution system via firefly algorithm. In: Proceedings of 2012 IEEE International Power Engineering and Optimization Conference. Melaka: IEEE, 2012. $84-89$

28 Jiang Y. Building Energy Efficiency Technology and Application (in Chinese). Beijing: China Architecture Building Press, 2008 [江亿. 天然气热电冷联供技术及应用. 北京: 中国建筑工业出版社, 2008]

29 Yang Y. Optimal configuration and comprehensive evaluation of distributed CCHP system in southern China (in Chinese). Dissertation for Master Degree. Beijing: Institute of Engineering Thermal Physics, University of the Chinese Academy of Sciences, 2013 [杨允. 南 方地区分布式供能系统优化配置与综合评价研究. 硕士学位论文. 北京: 中国科学院大学工程热物理研究所, 2013] 
Summary for “多能源耦合系统的运行优化与改进分析”

\title{
Operation optimization and improvement analysis of multi-energy system
}

\author{
ZHANG Tao, ZHU Tong", CHEN Shang \& WU Zhu \\ School of Mechanical Engineering, Tongji University, Shanghai 201804, China \\ * Corresponding author, E-mail: zhu_tong@tongji.edu.cn
}

With the ongoing economic development, the energy demands increase constantly in China. However, the environmental pollution and energy consumption conflict with each other. The traditional combined cooling heating and power (CCHP) system combined with renewable energy is becoming a key point of pelples's discussion in recent years because of its clean, efficient, reproducible and other prominent advantages. In this paper, an operation optimization model for the energy system coupled of the photovoltaic system, the distributed energy system, energy storage system and the heat pump system is introduced. The cool-storage technology is applied in this construction object. Therefore, the cooling energy can be supplied through cool-storage equipment in the cold load peak time. Firstly, a regional multi-energy system is introduced and the running characteristics of the multi-energy system were analyzed considering the power consumption of main equipment and the optimal operation cost which was objective function. This main generator unit includes two ICE which the generating capacity is $625 \mathrm{~kW}$. The part-load characteristic of ICE, heat recovery boiler, the receiver refrigerator are demonstrated for the multi-energy operation model. Secondly, an intelligent optimization algorithm (firefly algorithm) is introduced to solve the operation parameters based on the multi-energy model, and the system operation parameters were obtained according to different typical daily load demand. The simulation program is compiled in Matlab, and the population size of firefly algorithm is 50, maximum number of iterations is 200 and the number of convergence is about 120. Moreover, the operating cost and the equipment contribution in typical days are showed in the results to analysis the running characteristics of multi-energy system. In the last, a case study is introduced to demonstrate the feasibility of the firefly algorithm and the aim of the economic analysis is to verify the effectiveness of improvement measures. The main power source includes power grid and ICE generator. The main cooling source includes screw type refrigerator, absorption refrigerator and cool-storage. The heating source includes ground source heat pump which runs all days on account of its less operating cost, gas-fired boiler and heat recovery boiler. The annual operation cost is about 11.77 millon yuan when the capacity of ICE is $2000 \mathrm{~kW}$, and is reduced $6.1 \%$ compared with original design. A ratio of electricity buy-back price to electricity price is introduced to increase the economic of the multi-energy. therefore, there are some benefits of sell electricity to back power grid. Moreover, The annual operation cost is about 11.12 millon yuan when the capacity of ICE is $2500 \mathrm{~kW}$ and the ratio of electricity buy-back price to electricity price is 0.8 , and is reduced $11.3 \%$ compared with original design. The static payback period is about 4.43 years. In summary, the improvement analysis to further reduce the system operation cost was completed according to change the original design scheme. The results show that the optimization algorithm based on the firefly algorithm could more accurate solve multi-energy system running status parameters optimization, the typical day energy cost could be significantly reduced by increasing the capacity of the gas internal combustion engine units and the reasonable feed-in tariff policy could further reduce the operation cost.

multi-energy system, operation optimization, firefly algorithm, distributed energy system

doi: 10.1360/N972017-00635 